\title{
Dynamic changes in Mcl-1 expression regulate macrophage viability or commitment to apoptosis during bacterial clearance
}

\author{
Helen M. Marriott, ${ }^{1}$ Colin D. Bingle, ${ }^{1}$ Robert C. Read, ${ }^{1}$ Karen E. Braley, ${ }^{2}$ Guido Kroemer, ${ }^{3}$ \\ Paul G. Hellewell, ${ }^{4}$ Ruth W. Craig,, ${ }^{2}$ Moira K.B. Whyte, ${ }^{1}$ and David H. Dockrell ${ }^{1}$ \\ 1Division of Genomic Medicine, University of Sheffield, Sheffield, United Kingdom. 2Department of Pharmacology and Toxicology, \\ Dartmouth Medical School, Hanover, New Hampshire, USA. ${ }^{3}$ Centre National de la Recherché Scientifique, UMR 8125 , \\ Institut Gustave Roussy, Villejuif, France. ${ }^{4}$ Division of Clinical Sciences North, University of Sheffield, Sheffield, United Kingdom.
}

\begin{abstract}
Macrophages are critical effectors of bacterial clearance and must retain viability, despite exposure to toxic bacterial products, until key antimicrobial functions are performed. Subsequently, host-mediated macrophage apoptosis aids resolution of infection. The ability of macrophages to make this transition from resistance to susceptibility to apoptosis is important for effective host innate immune responses. We investigated the role of Mcl-1, an essential regulator of macrophage lifespan, in this switch from viability to apoptosis, using the model of pneumococcal-associated macrophage apoptosis. Upon exposure to pneumococci, macrophages initially upregulate Mcl-1 protein and maintain viability for up to 14 hours. Subsequently, macrophages reduce expression of full-length Mcl-1 and upregulate a 34-kDa isoform of Mcl-1 corresponding to a novel BH3-only splice variant, $\mathrm{Mcl}-\mathrm{1}_{\text {Exon-1}}$. Change in expression of Mcl-1 protein is associated with mitochondrial membrane permeabilization, which is characterized by loss of mitochondrial inner transmembrane potential and translocation of cytochrome $c$ and apoptosis-inducing factor. Following pneumococcal infection, macrophages expressing fulllength human Mcl-1 as a transgene exhibit a delay in apoptosis and in bacterial killing. Mcl-1 transgenic mice clear pneumococci from the lung less efficiently than nontransgenic mice. Dynamic changes in Mcl-1 expression determine macrophage viability as well as antibacterial host defense.
\end{abstract}

\section{Introduction}

Macrophages are the resident professional phagocytes in noninflamed tissues and survive in situ for extended periods of time (1). In keeping with this phenotype, macrophages exhibit inherent resistance to apoptosis and maintain viability in the presence of proapoptotic factors (2). Bacterial infection is a potent stimulus for apoptosis, and microbial virulence factors inhibit macrophage viability to impede antibacterial defenses (3). Surprisingly, macrophage apoptosis can also facilitate immune responses by limiting bacterial replication (4). This apparent paradox suggests that optimal macrophage antibacterial responses require transient maintenance of cell viability in the face of proapoptotic stimuli, followed by apoptosis induction for maximal bacterial clearance and minimal immune-mediated tissue injury. The control of this switch in macrophage phenotype is therefore an essential regulator of innate host defense.

The molecular basis of macrophage resistance to apoptosis includes expression of antiapoptotic Bcl-2 family members, among which Mcl-1 predominates in differentiated human macrophages (2). Mcl-1 was first identified as a molecule that is upregulated

\footnotetext{
Nonstandard abbreviations used: AIF, apoptosis-inducing factor; AM, alveolar macrophage; BMDM, bone marrow-derived macrophage; EGFP, enhanced GFP; JC-1, 5,5',6,6'-tetrachloro-1,1',3,3'-tetraethylbenzimidazolocarbocyanine iodide; $\Delta \psi_{\mathrm{m}}$, mitochondrial inner transmembrane potential; MDM, monocyte-derived macrophage; MG-132, carbobenzoxy-L-leucyl-L-leucyl-L-leucinal; MMP, mitochondrial membrane permeabilization; zVADfmk, $N$-benzyloxycarbonyl-Val-Ala-Asp ( $O$-methyl) fluoromethyl ketone.

Conflict of interest: The authors have declared that no conflict of interest exists.

Citation for this article: J. Clin. Invest. 115:359-368 (2005).

doi:10.1172/JCI200521766
}

during differentiation of immature myeloid cells along the monocyte/macrophage pathway (5). It is unique among antiapoptotic members of the Bcl-2 family in that it is an early-response gene that can be rapidly induced and turned over $(6,7)$. Mcl-1 is expressed during critical transitions in cell phenotype and protects against apoptosis during the initial steps of differentiation, exposure to growth stimuli, or the rapid response to potentially cytotoxic stimuli including chemotherapeutic agents, UV irradiation, and calcium ionophores $(5,8,9)$. The importance of Mcl-1 to macrophage survival has been confirmed in Mcl-1 transgenic mice and by antisense inhibition in human macrophages $(2,10)$.

Antiapoptotic Bcl-2 family members exert their effect in part by maintaining mitochondrial homeostasis and inhibiting mitochondrial membrane permeabilization (MMP) (11). Mcl-1 is found in mitochondrial-enriched cell fractions and can heterodimerize with proapoptotic Bcl-2 family members, such as Bax and Bak, to prevent MMP $(8,12)$. Loss of Mcl-1 is an apical event in the apoptotic cascade, as illustrated by its downregulation during UV-induced or adenoviral protein E1A-induced apoptosis $(12,13)$. Mcl-1 is subject to differential splicing, resulting in the generation of proapoptotic splice variants $(14,15)$. Mcl-1 is, therefore, an ideal regulator of cell viability during rapidly evolving conditions of cell stress and could allow the dynamic changes in cell viability required during bacterial infection.

The most extensively studied model of host-mediated macrophage apoptosis involves the chronic intracellular pathogen Mycobacterium tuberculosis, in which apoptosis limits bacterial replication and, conversely, intracellular replication is prolonged by inhibiting apoptosis $(4,16)$. Mcl-1 expression is upregulated in macrophages by $M$. tuberculosis and inhibits host-mediated 
A

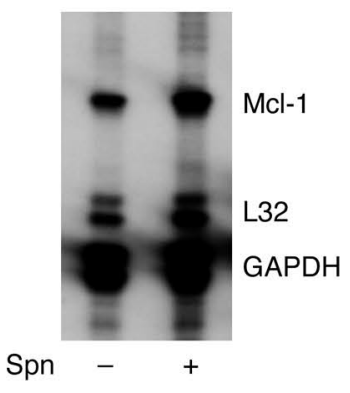

B
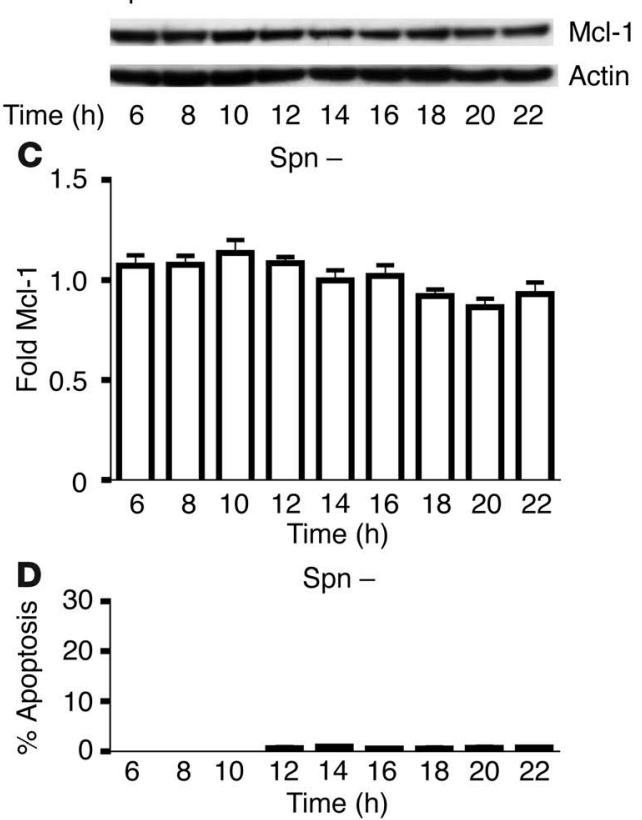

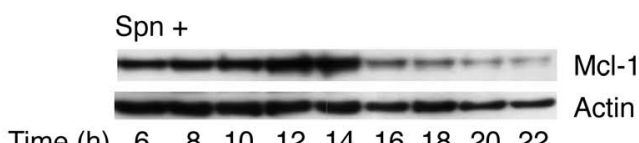

Time (h) $6 \quad 8 \quad 10 \quad 12 \quad 14 \quad 16 \quad 18 \quad 2022$ Spn +
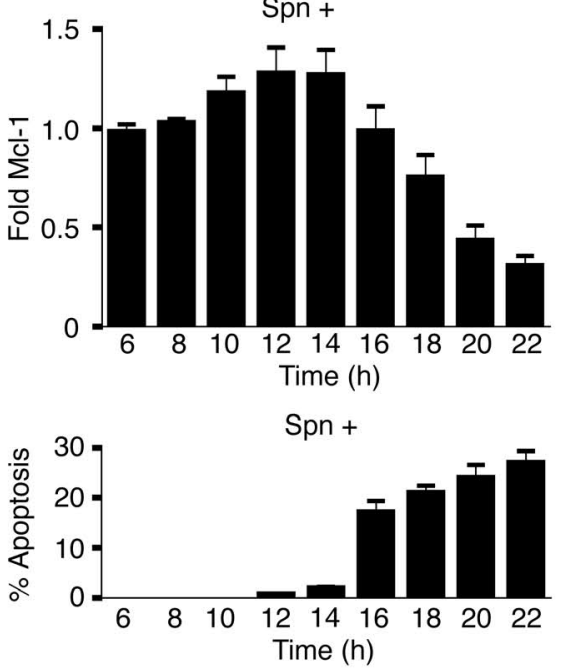

Figure 1

Mcl-1 protein levels demonstrate biphasic variation in association with pneumococcal infection of macrophages. (A) Total RNA was extracted from MDMs 20 hours after mock infection (Spn-) or infection with pneumococci (Spn+), and Mcl-1 mRNA was detected by RNase protection assay. The results presented are typical of 5 donors tested, but the duration of mRNA elevation varied among donors. L32 and GAPDH served as loading controls. (B) Western blot of total protein from MDMs at the indicated time points after infection, probed with anti-Mcl-1 and antiactin antibodies. Data are representative of 7 independent experiments. (C) Mcl-1 band density from a series of MDMs as in B, quantified by densitometry $(n=7) . P<0.05$, Mcl-1 at 6 hours vs. 12 hours; $P<0.001,6$ hours vs. 20 hours; Student's $t$ test. (D) Levels of nuclear apoptosis in the same series of MDMs as in C. $P<0.001$, apoptosis at 6 hours vs. 16 hours, Student's $t$ test.

apoptosis, hence promoting mycobacterial survival and chronic intracellular persistence (16). We have characterized a different model of host-mediated macrophage apoptosis involving Streptococcus pneumoniae (the pneumococcus). This model does not exhibit chronic intracellular persistence of bacteria $(17,18)$. Instead, bacteria are effectively eliminated through a response that proceeds in 2 phases: an initial period of macrophage viability and intracellular bacterial killing, followed after 14-16 hours by apoptosis induction and clearance of bacteria. Inhibition of the latter phase of apoptosis induction reduces bacterial clearance in vitro and in vivo $(17,18)$. This model therefore allows analysis of both phases of the macrophage response to bacterial infection.

We used a model of pneumococcal-associated host-mediated macrophage apoptosis to test the hypothesis that Mcl-1 is critical to both initial maintenance of cell viability and subsequent induction of apoptosis. Mcl-1 was upregulated in association with the retention of cell viability after initial bacterial phagocytosis. Mcl-1 was subsequently downregulated, and a protein of approximately $34 \mathrm{kDa}$ with an electrophoretic mobility corresponding to a novel proapoptotic $\mathrm{BH} 3$-only splice variant $\mathrm{Mcl}-1_{\text {Exon- } 1}$ was upregulated, during the initiation of MMP and induction of apoptosis. These changes in Mcl-1 expression have a role in the response to infection, as macrophages from transgenic mice overexpressing Mcl-1 demonstrated delayed MMP and impaired bacterial clearance in vitro. Mcl-1 transgenic mice demonstrated less effective clearance of bacteria from the lung in vivo. These findings demonstrate the importance of an Mcl-1-regulated switch from macrophage viability to apoptosis for optimal host defense.

\section{Results}

A biphasic response in monocyte-derived macrophage expression of Mcl-1 protein is observed after phagocytosis of pneumococci. We have previously demonstrated that after phagocytosis of pneumococci, an initial period of macrophage viability is followed by apoptosis (17). To investigate whether changes in Mcl-1 transcription occurred in macrophages in response to exposure to pneumococci, we performed RNase protection assays. Mcl-1 mRNA was upregulated upon pneumococcal infection (Figure 1A). To correlate Mcl-1 protein expression with macrophage survival and death, we serially measured Mcl-1 expression in monocyte-derived macrophages (MDMs) from 7 donors 6-20 hours after infection. To take account of the interdonor variability inherent to MDMs, both a representative Western blot and a summary graph depicting protein expression for all 7 donors are presented, and protein expression is compared with the number of apoptotic cells at each time point (Figure 1, B-D). Mcl-1 levels were upregulated in infected MDMs compared with mock-infected MDMs for as long as 14 hours after infection. After this time point, all donors demonstrated a progressive decline until Mcl-1 levels dropped below those observed in mock-infected MDMs. The time at which this occurred varied among donors, and the time at which Mcl-1 started to decline best predicted the onset of apoptosis in infected cultures (Figure 1D). To examine whether the alterations in Mcl-1 expression were part of a general alteration in antiapoptotic $\mathrm{Bcl}-2$ proteins, we also examined $\mathrm{Bcl}-\mathrm{x}_{\mathrm{L}}$ but found it to be unaltered (see data in Figure 4A).

Decreased Mcl-1 protein expression is associated with MMP during pneumococcal-associated MDM apoptosis. MMP, inhibited by antiapoptotic Bcl-2 family members, occurs upstream of caspase 
A
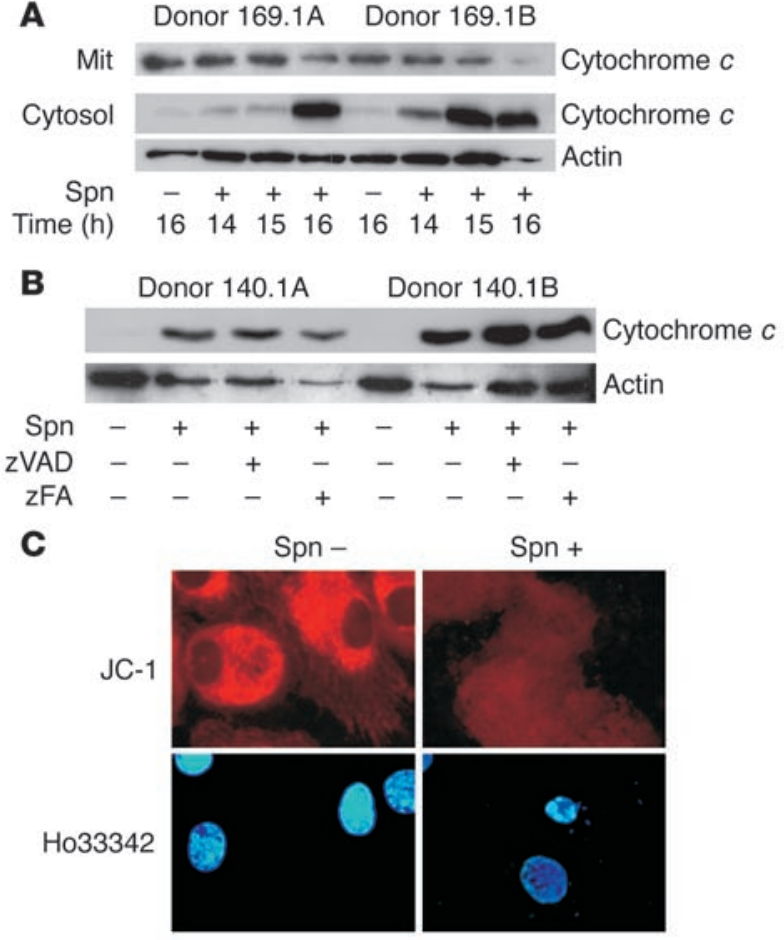

D
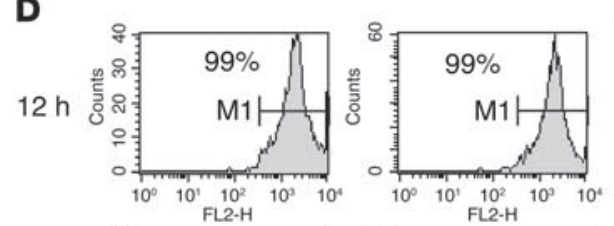

$\mathbf{E}$

$14 \mathrm{~h}$
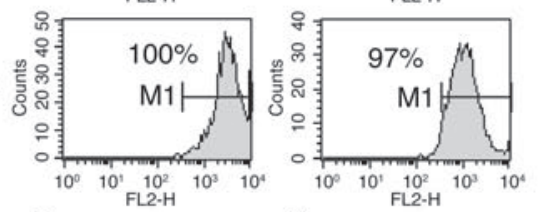

$15 \mathrm{~h}$
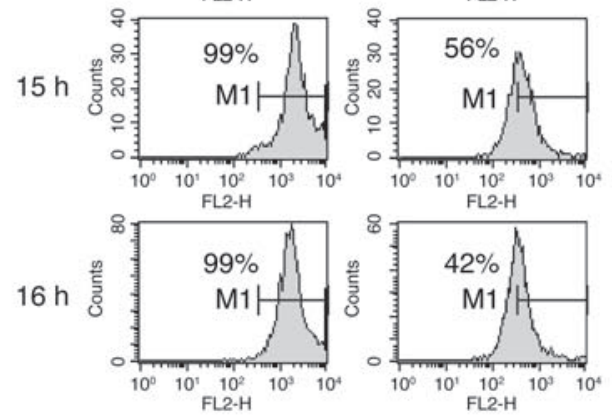

\section{Figure 2}

Mitochondrial features of apoptosis are observed in macrophages infected with pneumococci. (A) Western blot of the cytosolic and mitochondrial (Mit) fractions from MDMs at the indicated time points after infection with pneumococci or mock infection probed with anti-cytochrome $c$ and anti-actin antibodies. Results are derived from 2 donors and are representative of 2 independent experiments. (B) Western blot of the cytosolic fractions from MDMs 20 hours after infection in the presence (+) or absence (-) of zVADfmk or N-benzyloxycarbonyl-Phe-Ala fluoromethyl ketone (zFA) probed with anti-cytochrome $c$ and anti-actin antibodies. Results are derived from 2 donors and are representative of 2 independent experiments. (C) MDMs were stained with JC-1 and Hoechst 33342 (Ho33342) 20 hours after infection. Loss of $\Delta \psi_{\mathrm{m}}$ is represented as a loss of punctate red staining, and nuclear morphology is detected by Hoechst 33342 staining. Data are representative of 3 donors. (D) Histograms representing $\Delta \psi_{\mathrm{m}}$ of MDMs at the indicated time points after infection, stained with JC-1. The percentage of cells with preservation of $\Delta \psi_{\mathrm{m}}$ (indicated by the M1 marker) is shown. Results are representative of 3 independent experiments. (E) Western blot of nuclear fractions from MDMs 20 hours after infection, probed with anti-AIF and Histone $\mathrm{H} 1$ antibodies. Data are representative of 3 independent experiments.

activation in many pathways of programmed cell death (11). MMP is characterized by loss of mitochondrial inner transmembrane potential $\left(\Delta \psi_{\mathrm{m}}\right)$ and the mitochondrial release of intermembrane proteins such as cytochrome $c$ and apoptosis-inducing factor (AIF) $(11,19)$. Cytochrome $c$ translocation was observed in infected MDMs 14-16 hours after infection, and actin degradation was a feature of the late stages of apoptosis but occurred after translocation of cytochrome $c$ and downregulation of Mcl-1 (Figure 2A). To exclude the possibility that mitochondrial features of apoptosis are a consequence of a caspase amplification loop and occur downstream of caspase activation (11), we determined the relationship of mitochondrial permeability transition to caspase activation. Treatment with a pancaspase inhibitor, $N$-benzyloxycarbonyl-Val-Ala-Asp ( $O$-methyl) fluoromethyl ketone (zVADfmk), did not suppress cytochrome $c$ translocation (Figure $2 \mathrm{~B}$ ), suggesting that it occurs independently of caspase activation. Loss of $\Delta \psi_{\mathrm{m}}$ is also a feature of pneumococcalassociated macrophage apoptosis and is detected prior to nuclear fragmentation (Figure 2C). Loss of $\Delta \psi_{\mathrm{m}}$ showed the same kinetics as cytochrome $c$ translocation (Figure 2D) and was not prevented by caspase inhibition (data not shown). AIF has been implicated in pneumococcal-associated neuronal cell death (20). Nuclear fractions from pneumococcal-infected (but not mock-infected) MDMs contained AIF and exhibited histone $\mathrm{H} 1$ degradation (Figure 2E).
The earliest features of MMP were coincident with the initial decline in peak Mcl-1 protein expression, emphasizing that downregulation of Mcl-1 was an early event in apoptosis induction.

Production of a novel BH3-only isoform of Mcl-1 is a feature of pneumococcal-associated macrophage apoptosis. Analysis of Mcl-1 protein expression in MDMs after pneumococcal infection revealed induction of an additional band of approximately $34 \mathrm{kDa}$ on Western blots probed with anti-Mcl-1 antibody (Figure 3A). In the series of MDMs derived from 7 different donors, expression of the band of approximately $34 \mathrm{kDa}$ occurred from 12 hours after infection and prior to onset of MMP. Treatment of infected MDM cultures with the transcription inhibitor actinomycin D blocked expression of this protein (data not shown). We and others have shown that altered splicing of the Mcl-1 gene can result in production of a BH3-only proapoptotic protein $(14,15)$. The band of approximately $34 \mathrm{kDa}$ detected in SDS-PAGE from infected MDMs exhibited a higher electrophoretic mobility than the in vitro translation product of the $\mathrm{Mcl}-1_{\mathrm{S} / \Delta \mathrm{TM}}$ splice variant, but a migration similar to that of the product of the Mcl- $1_{\text {Exon- } 1}$ splice variant (Figure $3 \mathrm{~B}$ ). Mcl- $1_{\text {Exon- } 1}$ is the product of an mRNA that retains intron 1 and contains 230 amino acids that are shared with full-length Mcl-1 and $\mathrm{Mcl}-1_{\mathrm{S} / \Delta \mathrm{TM}}$ plus an additional 4 amino acids (Figure $3 \mathrm{C}$ ) (C.D. Bingle, unpublished observations). Upregulation of this isoform in 


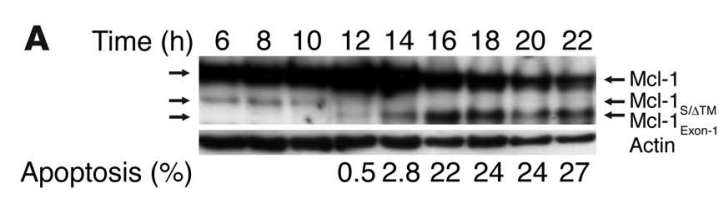

C

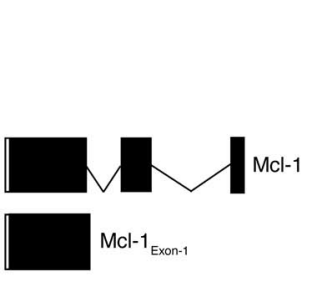

D

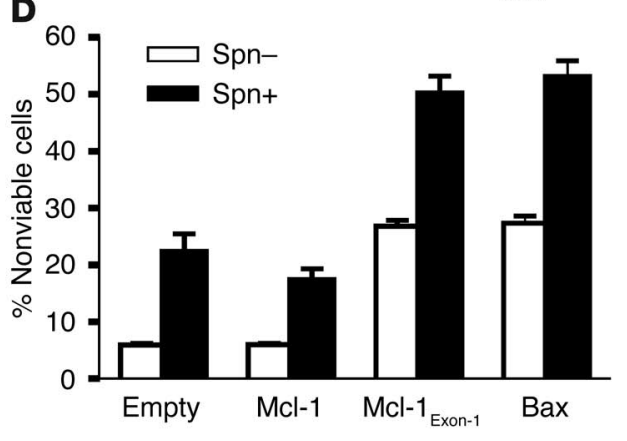

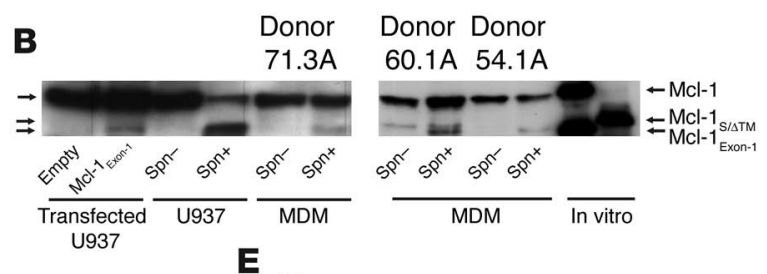

E

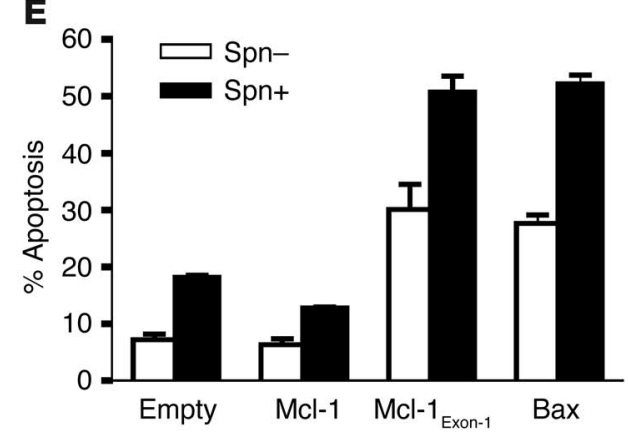

Figure 3

Pneumococcal infection of macrophages results in increased expression of a proapoptotic Mcl-1 isoform. (A) Western blot of total protein from MDMs at the indicated time points after infection with pneumococci, probed with anti-Mcl-1 and anti-actin antibodies showing the appearance of the band of approximately $34 \mathrm{kDa}$. The donor is representative of 1 of the series in Figure $1 \mathrm{C}$. The mean levels of nuclear apoptosis at each time point for this donor are indicated. (B) Western blot of total protein from U937 cells or MDMs (3 donors in 3 separate experiments) 20 hours after infection with pneumococci or mock infection and U937 cells transfected with Mcl- $1_{\text {Exon-1 }}$ or empty vector probed with anti-Mcl-1 antibody, with in vitro translation of $\mathrm{Mcl}-1, \mathrm{Mcl}-1_{\mathrm{S} / \Delta \mathrm{TM}}$, and $\mathrm{Mcl}-1_{\text {Exon-1 }}$ as standards. (C) Genomic organization of Mcl-1 and Mcl-1 Exon-1 $_{1}$ The filled regions represent the coding region of the 2 transcripts. (D) Percentage of nonviable infected or mock-infected U937 cells 16 hours after nucleofection with vectors containing full-length $\mathrm{Mcl}-1, \mathrm{Mcl}-1_{\mathrm{Exon}-1}$, Bax, or empty vector. Results represent the analysis of 3 separate experiments. $P<0.05$, Spn- Mcl-1 vs. Spn- Mcl-1 Exon-1; $P<0.05$, Spn+ Mcl-1 vs. Spn+ Mcl-1Exon-1; Student's paired $t$ test. (E) Percentage of EGFP-positive cells demonstrating features of apoptosis after DAPI staining in the same experiments as in D. $P<0.05$, Spn- Mcl- 1 vs. Spn- Mcl- $1_{\text {Exon-1 }} ; P<0.05$, Spn+ $\mathrm{Mcl}-1$ vs. Spn+ Mcl-1 Exon-1; $P<0.05$, Spn+ empty vector vs. Spn+ Mcl-1; Student's paired $t$ test.

response to infection was confirmed in the human monocytic cell line U937, where transfection with a construct encoding Mcl-1 $1_{\text {Exon-1 }}$ or infection with pneumococci resulted in expression of an identical $34-\mathrm{kDa}$ protein that was distinct from $\mathrm{Mcl}-1_{\mathrm{S} / \Delta \mathrm{TM}}$ (Figure $3 \mathrm{~B}$ ). The proapoptotic function of $\mathrm{Mcl}-1_{\text {Exon- } 1}$ was demonstrated in mockinfected and pneumococcal-infected U937 cells, where transfection with a construct encoding Mcl- $1_{\text {Exon- } 1}$ both decreased cell viability and increased apoptosis (Figure 3, D and E). Levels of apoptosis in cells transfected with Mcl-1 $1_{\text {Exon-1 }}$ were comparable to those in cells transfected with the proapoptotic Bcl-2 family member Bax. Furthermore, $\mathrm{Mcl}-1$ transfection significantly decreased the percentage of apoptotic cells among the population positive for enhanced GFP (EGFP) after pneumococcal infection, as compared with transfection with empty vector. These results demonstrate the interplay between full-length Mcl-1 and Mcl-1 $1_{\text {Exon-1. }}$.

Mcl- 1 contains caspase cleavage sites, and 4 caspase cleavage products of $17-28 \mathrm{kDa}$ in size have been described (21). However, treatment with zVADfmk failed to alter the abundance of Mcl-1 $1_{\text {Exon-1 }}$ (Figure 4A). Similarly, caspase inhibition did not prevent the decrease in Mcl-1 expression associated with pneumococcal infection (data not shown). To exclude the possibility that other proteases active in infected MDMs might generate the isoform of Mcl-1 that was approximately $34 \mathrm{kDa}$, we treated cultures with inhibitors of cysteine or serine proteases, but none resulted in decreased expression of the protein (data not shown). Subcellular fractionation demonstrated that Mcl-1 $1_{\text {Exon-1, }}$ like Mcl-1, was localized to the mitochondrial membrane, despite loss of the carboxyl hydrophobic tail associated with mitochondrial localization of the full-length protein (6) (Figure 4B). A small amount of Mcl-1 was also apparent in the cytosolic fraction of mock-infected cells, but this became undetectable by the time cytochrome $c$ first translocated to the cytoplasm. Mcl-1 downregulation can be a feature of cell stress pathways in which p53 accumulates (12). To determine whether Mcl-1 $1_{\text {Exon-1 }}$ expression is also induced by stimuli that induce apoptosis by downregulating full-length Mcl-1, we examined the effect of UV treatment on MDMs (13). As expected, UV irradiation induced nuclear fragmentation in MDMs (Figure 4C) and p53 upregulation (Figure 4D). Although UV irradiation was associated with Mcl-1 downregulation, there was no evidence of production of Mcl-1 $1_{\text {Exon-1 }}$ at any time point following UV exposure, suggesting that production of Mcl- $1_{\text {Exon- } 1}$ is not a feature of UV-mediated MDM apoptosis.

Proteasome-mediated degradation of Mcl-1 occurs in pneumococcalassociated macrophage apoptosis. Mcl-1 is degraded by the ubiquitinproteasome pathway (13). Mcl-1 levels were increased in cell lysates following treatment with the proteasome inhibitor carbobenzoxy-L-leucyl-L-leucyl-L-leucinal (MG-132), regardless of whether they were mock-infected or infected (Figure 5A). At 20 hours after infection, proteasome inhibition with MG-132 increased levels of Mcl-1, although not to the same level as observed in mock-infected cultures, suggesting that proteasomal degradation by this pathway is not the sole explanation for loss of Mcl-1 protein. However, the increased levels of Mcl-1 after proteasome inhibition were associated with decreased cytochrome $c$ translocation to the cytosol in pneumococcalinfected MDMs (Figure 5B). Furthermore, proteasome inhibition in pneumococcal-infected MDMs decreased nuclear features of apoptosis (Figure 5C). Although proteasome inhibition can reduce degradation of multiple antiapoptotic proteins in addi- 
A

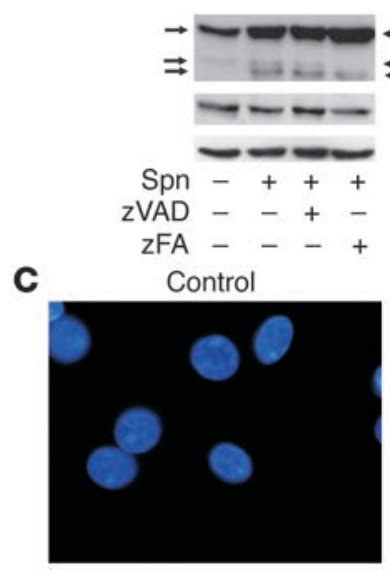

B

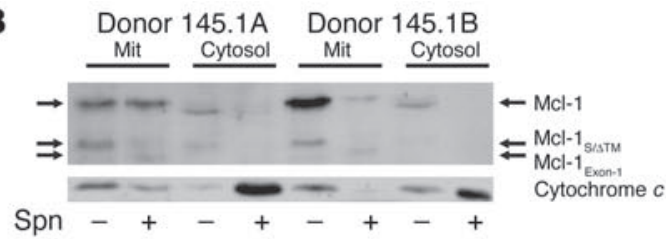

D

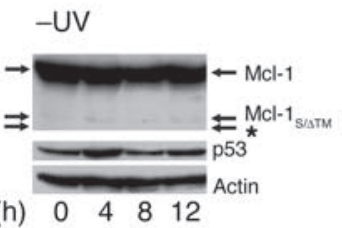

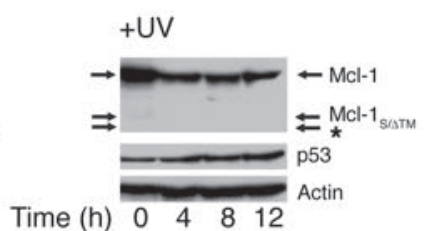

Figure 4

Mcl-1 Exon-1 expression is an upstream event in pneumococcal-associated but not UV-associated macrophage apoptosis. (A) Western blot of total protein from MDMs 20 hours after infection in the presence (+) or absence (-) of zVADfmk (zVAD) or N-benzyloxycarbonyl-Phe-Ala fluoromethyl ketone (zFA), probed with anti-Mcl-1, anti-Bcl- $\mathrm{x}_{\mathrm{L}}$, and anti-GAPDH antibodies. Data is representative of 4 donors. (B) Western blot of membrane and nuclear fractions from MDMs 20 hours after infection, probed with anti-Mcl-1 antibody. Data represent 2 donors and 3 independent experiments. (C) Nuclear morphology, detected by DAPI staining, in control MDMs (left panel) or MDMs 12 hours after UV irradiation (right panel). Levels of apoptosis were $1 \%$ in control MDMs and 34\% in UV-irradiated MDMs. (D) Western blot of total protein from MDMs at the indicated time points after UV irradiation, probed with anti-Mcl-1, anti-p53, and anti-actin antibodies. The asterisks mark the expected positions of Mcl- $1_{\text {Exon-1. }}$. Data are representative of 3 donors.

tion to Mcl-1 (13), these results suggest that proteasome-mediated degradation of Mcl-1 contributes to the loss of macrophage viability following phagocytosis of pneumococci.

Bone marrow-derived macrophages from Mcl-1 transgenic mice demonstrate decreased apoptosis after pneumococcal infection. To further test the concepts that $\mathrm{Mcl}-1$ contributes to the switch in macrophage phenotype and that abrogating this switch might negatively affect clearance of bacteria, we performed experiments with bone marrow-derived macrophages (BMDMs) derived from Mcl-1 transgenic mice or their nontransgenic littermates (10). Lysates from transgenic mice, but not those from nontransgenic mice, reacted with an anti-human Mcl-1 antibody that does not detect murine Mcl-1 (Figure 6A). Transgenic BMDMs exposed to pneumococci also demonstrated preservation of $\Delta \psi_{\mathrm{m}}$ (Figure 6, B and C), decreased translocation of cytochrome $c$ from the mitochondria (data not shown), and fewer nuclear changes indicative of apoptosis than did nontransgenic BMDMs (Figure 6, $\mathrm{D}$ and $\mathrm{E}$ ). Pneumococcal infection of BMDMs resulted in a decrease in expression of the Mcl-1 transgene (Figure $6 \mathrm{~F}$ ). $\mathrm{Mcl}-1_{\text {Exon- } 1}$ protein

\section{Figure 5}

Proteasome-mediated degradation of $\mathrm{Mcl}-1$ is associated with pneumococcal-associated macrophage apoptosis. (A) Western blot of total protein obtained from MDMs 12 hours and 20 hours after mock infection or infection with pneumococci in the presence or absence of the proteasome inhibitor MG-132, probed with anti-Mcl-1 and antiactin antibodies. Data are representative of 4 donors in 2 independent experiments. (B) Western blot of the cytosolic fractions from MDMs 20 hours after infection in the presence or absence of MG-132 probed with anti-cytochrome $c$ and anti-actin antibodies. Results are representative of 3 donors from 2 independent experiments. (C) MDMs were incubated in the presence or absence of MG-132, and nuclear features of apoptosis were recorded 20 hours after infection. Results were obtained from 6 donors tested in duplicate. Dunn's multiple comparison test: MDMs infected with pneumococci without MG-132 vs. with MG-132, $P<0.05$. Data are representative of 3 experiments. was also found in the lysates of BMDMs from Mcl-1 transgenic mice after pneumococcal infection but not after mock infection (data not shown). In keeping with this decreased expression of Mcl-1 after infection and the rapid turnover of Mcl-1 $(6,7)$, the decreased apoptosis observed in transgenic BMDMs represented a delay in apoptosis rather than a sustained inhibition, similar to previous observations with Mcl-1 $(8,10)$. The preservation of $\Delta \psi_{\mathrm{m}}$ apparent at 14-18 hours was no longer detectable at 20 hours after infection, and the lack of nuclear apoptosis at 20 hours disappeared by 48 hours after infection in transgenic BMDMs (data not shown).

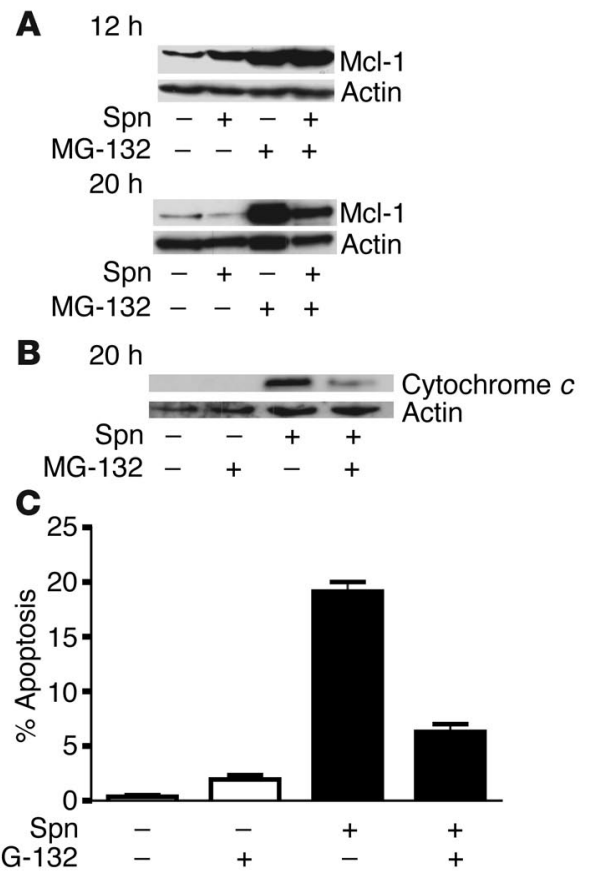


A

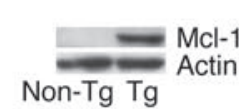

B
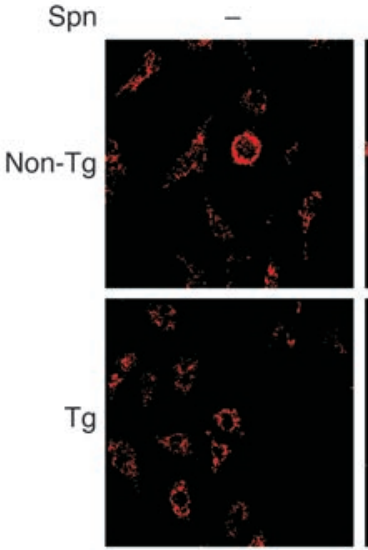

D Spn
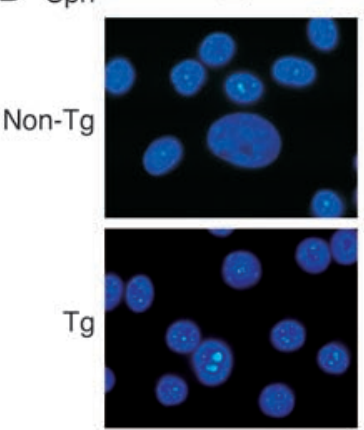

$+$

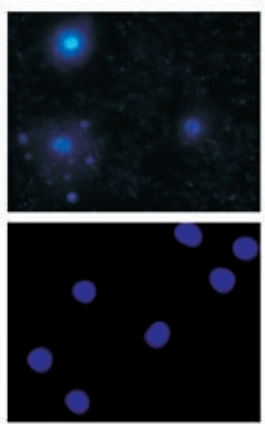

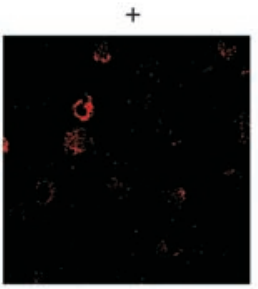

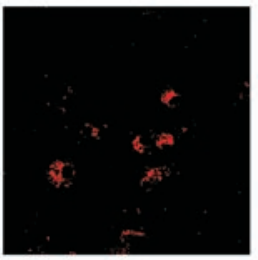

E
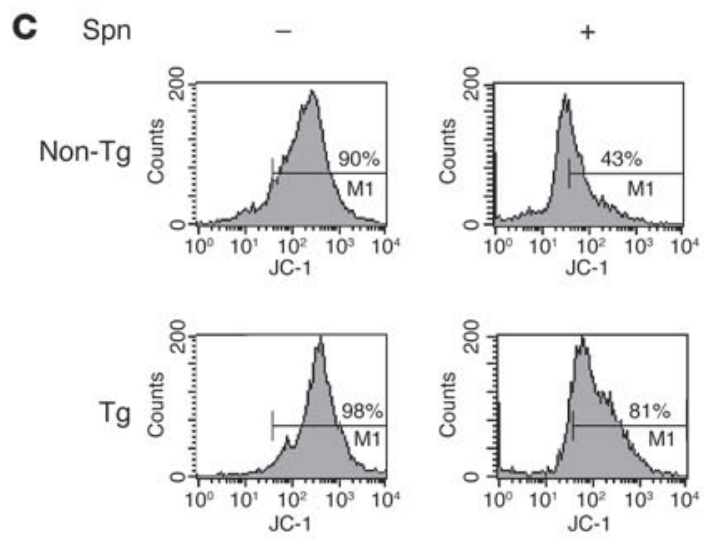

$\mathbf{F}$
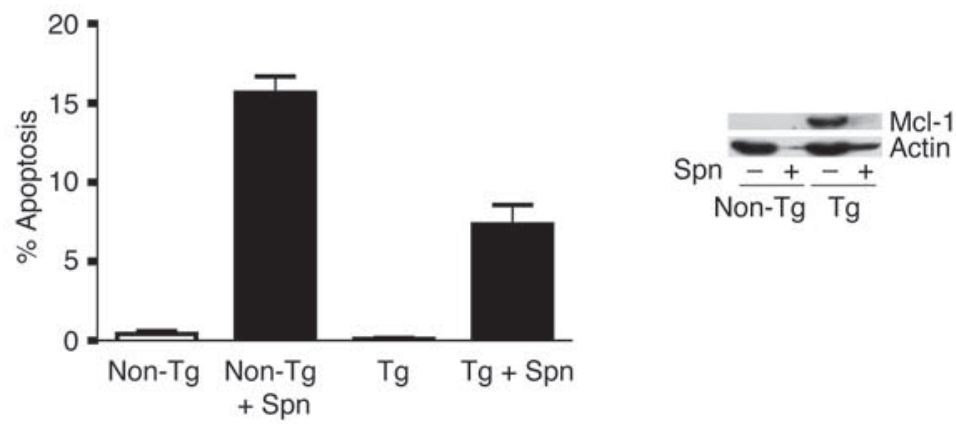

Figure 6

Macrophages from Mcl-1 transgenic mice have delayed apoptosis. (A) Western blot of total protein extracted from BMDMs from nontransgenic mice (Non-Tg) and mice transgenic for human Mcl-1 (Tg) and probed with anti-human Mcl-1 and anti-actin antibodies. (B) BMDMs from nontransgenic and transgenic mice were stained with JC-1 14 hours after mock infection or infection with pneumococci. Data are representative of 3 experiments. (C) Histograms representing $\Delta \psi_{\mathrm{m}}$ of BMDMs from nontransgenic and transgenic controls 14 hours after infection, stained with JC-1. Results are representative of 3 independent experiments. (D) Nuclear morphology, detected by DAPI staining, in BMDMs from nontransgenic and transgenic mice 20 hours after infection. (E) Nuclear features of apoptosis were recorded from BMDMs from transgenic or nontransgenic controls 20 hours after infection. $P<0.05$, BMDMs infected with pneumococci from transgenic vs. nontransgenic, Dunn's multiple comparison test. Results represent 3 experiments. (F) Western blot of total protein from BMDMs from nontransgenic and transgenic mice 20 hours after infection, probed with anti-human Mcl-1 and anti-actin antibodies.

Clearance of pneumococci from the lung of Mcl-1 transgenic mice. We have previously documented that caspase activation not only contributes to apoptosis induction in macrophages following pneumococcal phagocytosis (22), but also enhances bacterial clearance (17). In association with delayed apoptosis in BMDMs from Mcl-1 transgenic mice, we also observed an increased viability of intracellular pneumococci (Figure 7A). We have previously described a mouse model of pulmonary pneumococcal infection in which apoptosis of alveolar macrophages (AMs) contributes to optimal bacterial clearance (18). Using this model, we instilled pneumococci into the lungs of transgenic or nontransgenic mice and measured apoptosis in AMs 14 hours after infection. Transgenic mice had significantly fewer apoptotic AMs (Figure 7, B and C) and significantly less efficient clearance of bacteria from the lungs (Figure 7D). All nontransgenic mice cleared $10^{4} \mathrm{CFU}$ of pneumococci from the lung, but at this dose, transgenic mice had detectable numbers of bacteria in the lung. In addition, all transgenic mice with more than $10^{2} \mathrm{CFU}$ in the lung developed bacteremia, a finding not seen in the nontransgenic mice. When the inocula of bacteria were increased to the threshold at which AM host defenses become overwhelmed, nontransgenic mice no longer cleared bacteria completely, but their mean bacterial colony count was still lower by a factor of greater than 1 on a log scale. For the small number of mice tested $(n=3-4)$, however, this result did not reach statistical significance. Overall, these results show that AM apoptosis is decreased in Mcl-1 transgenic mice after pneumococcal infection and that this is associated with a significant impairment of bacterial clearance.

\section{Discussion}

Mcl-1 protein expression regulates macrophage transition from resistance to susceptibility to apoptosis during pneumococcal infection. To contribute optimally to host defense, macrophages demonstrate biphasic susceptibility to apoptosis. Initial resistance to apoptosis in the face of proapoptotic host and microbial factors allows the macrophages to contribute to innate immunity $(3,23)$, but subsequent induction of apoptosis facilitates bacterial clearance and minimizes inflammation (18). The molecular events associated with this transition are unknown, and the present research has stemmed from our interest in identifying factors that regulate this switch in macrophage phenotype.

We focused on the model of host-mediated macrophage apoptosis during pneumococcal infection, because it is characterized by initial 
A
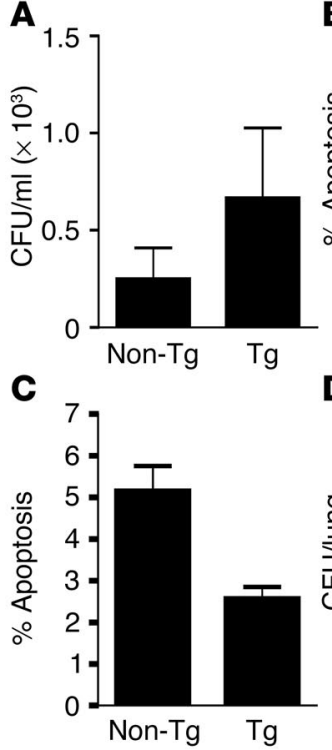

B

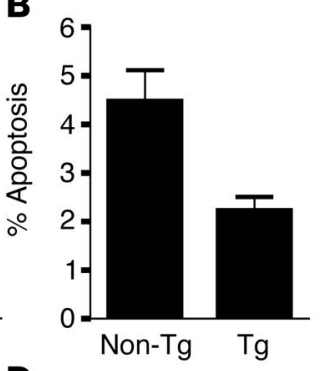

D

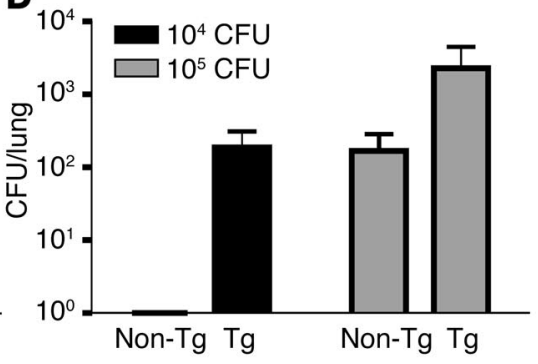

macrophage viability and subsequent macrophage apoptosis $(17,18)$. A tightly regulated biphasic pattern in macrophage susceptibility to apoptosis is essential for optimal killing of bacteria in this model. Pneumococci are innately resistant to phagocytosis and challenge the capacity of host defenses to clear bacteria successfully (24). Nevertheless, phagocytosis of opsonized bacteria by macrophages results in clearance of bacteria without intracellular persistence and is associated with macrophage apoptosis $(22,24)$. In contrast to mycobacteria, which tend to persist intracellularly $(25,26)$, the pneumococcal model of host-mediated macrophage apoptosis involves phagocytosis and acute bacterial killing without prolonged intracellular survival (22), enabling investigation of biphasic susceptibility to apoptosis.

Mcl-1 has been identified as a specific determinant of the resistance of macrophages to apoptosis and acts during periods of changing cell phenotype $(2,5)$. As shown in this work, upregulation of Mcl-1 mRNA was associated with sustained macrophage viability for at least 14 hours after exposure to pneumococci. As proof of concept, transfection of U937 cells with Mcl-1 decreased the level of apoptosis after pneumococcal infection. Furthermore, transgenic mice overexpressing Mcl-1 exhibited reduced apoptosis after pneumococcal infection, both in BMDMs in vitro and in AMs in vivo. Mcl-1 upregulation in macrophages is a feature of mycobacterial infection (16), and enhanced Mcl-1 gene transcription has been identified in brain endothelium during infection with the group B streptococcus, an organism closely related to the pneumococcus (27). It is plausible that Mcl-1 upregulation may be utilized by a broad range of cell types to prevent apoptosis during infection (28).

$\mathrm{Bcl}-2$ family proteins protect against apoptosis during conditions of increased cell stress, including nitrosative or oxidative stress $(29,30)$, which prevail in macrophages loaded with bacteria (31). Mice deficient in adenine nucleotide translocator 1 demonstrate oxidative stress and upregulate Mcl-1 (32). In the first 12-14 hours after phagocytosis of pneumococci, Mcl-1 aids maintenance of mitochondrial homeostasis despite increasing cell stress. Following this period of macrophage viability, Mcl-1 expression declines, and signs of MMP and apoptosis are observed. Even BMDMs from Mcl-1 transgenic mice exhibited only a delay in MMP. Mcl-1

\section{Figure 7}

Mcl-1 transgenic mice demonstrate decreased AM apoptosis and decreased bacterial clearance from the lung. (A) Intracellular killing assays were performed in BMDMs from $\mathrm{Mcl}-1$ transgenic and nontransgenic controls 14 hours after infection. Results are representative of 4 separate experiments. $P<0.05$, transgenic vs. nontransgenic; Wilcoxon signed rank test. Transgenic and nontransgenic mice received an intratracheal instillation of the indicated dose of pneumococci. (B and $\mathbf{C}$ ) Percentage apoptotic macrophages in bronchial alveolar fluid from transgenic vs. nontransgenic mice after instillation of $10^{4} \mathrm{CFU}$ pneumococci as assessed by (B) Annexin V/ToPro 3 staining and flow cytometry $(P<0.01$; $n=6-8)$ and (C) nuclear morphology on cytospins $(P<0.01 ; n=6-8)$. (D) Bacterial CFU in lungs of transgenic vs. nontransgenic mice (104 CFU, $P<0.05, n=6-8 ; 10^{5}$ CFU, $\left.P>0.05, n=3-4\right)$.

downregulation is a feature of cell stress pathways (13), and a DNA damage-response pathway that mediates apoptosis in association with Mcl-1 downregulation with enhanced Bak-Bax heterodimerization has recently been described in HeLa cells infected with adenovirus and contributes to antiviral host defense (12). Late decreases in Mcl-1 protein levels were the result of both translational downregulation, and active proteasomal degradation-cellular stress pathways may influence both these processes $(12,13)$. Increased protein degradation was a constant finding, whereas the timing of downregulation of mRNA was variable with some donors showing decreased mRNA only after 20 hours. The critical determinant of the onset of apoptosis induction in this model seemed to be the timing of initial decline of Mcl- 1 expression from peak levels rather than the time at which the absolute level of Mcl-1 fell below the level expressed in mock-infected cells. Nitrosative/oxidative stress can trigger apoptosis in DNA-damage pathways (33), and we have recently shown that NO production contributes to MMP in pneumococcal-associated macrophage apoptosis (34). Mcl-1 may be downregulated in response to cellular stresses such as nitrosative/ oxidative stress, nucleotide depletion, or accumulating DNA damage in macrophages loaded with pneumococci. It has been reported that accumulating nitrosative stress in cells initially does not induce apoptosis, as glycolytic ATP production maintains viability, but once this source of ATP production fails, oxidative stress supervenes and MMP results (35). Therefore the initial mitochondrial protection provided by Mcl-1 is likely to be overwhelmed by the combination of Mcl-1 downregulation and accumulating cell stress.

Generation of a novel BH3-only isoform of Mcl-1 is associated with macrophage apoptosis during pneumococcal infection. We documented upregulation of a novel isoform of Mcl-1 of approximately 34

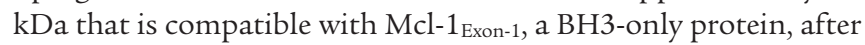
pneumococcal infection (but not after UV-induced apoptosis). Expression of this protein required active transcription but was not blocked by inhibitors of caspases, cathepsins, calpains, or serine proteases. Transfection of Mcl-1 Exon-1 $_{\text {into }}$ U937 cells confirmed that this protein was proapoptotic and had effects comparable to those of Bax in enhancing apoptosis in both the absence or the presence of pneumococcal infection. Alternative splicing is a feature of many $\mathrm{Bcl}-2$ proteins, and generation of an isoform in association with retention of an intron and generation of an open reading frame arising from a continuous genomic sequence has been described for the $\mathrm{Bcl}-\mathrm{x}$ isoform $\mathrm{Bcl}-\mathrm{x}_{\beta}(36)$. Although no specific binding partners of Mcl- $1_{\text {Exon-1 }}$ have been identified, it is interesting to note that another isoform of Mcl-1, Mcl- $1_{\mathrm{S} / \Delta \mathrm{TM}}(14)$, binds (and potentially neutralizes) full-length Mcl-1 (15). In addition, the localization of 
Mcl-1 $1_{\text {Exon- } 1}$ (which lacks the C-terminal transmembrane domain) to the mitochondrial fraction, suggests that it may interact with Bcl-2 family members already anchored to the mitochondrial membrane. Activated $\mathrm{BH} 3$-only proteins can bind antiapoptotic $\mathrm{Bcl}-2$ proteins releasing Bak/Bax from inhibition or directly activating Bak-Bax for MMP induction $(37,38)$.

Many Bcl-2 family isoforms have been identified and have been best characterized in BH3-only proteins such as Bim (39). However, these isoforms have been described in transformed cell lines or experiments involving cloning systems (40). There has been little information on the expression of Bcl-2 family isoforms during physiologic events such as infection or inflammation. The only reports concern the induction of 3 murine A1 isoforms in mice exposed to bacillus Calmette-Guerin, although these isoforms arise from transcription of 3 separate genes and do not arise from differential splicing (41). In contrast, in the present system, the differential splicing of a single gene would result in expression of an isoform that opposes the function of the full-length protein. Importantly, this isoform is not expressed by mock-infected macrophages, and its putative expression in infected cells occurs before Mcl-1 transcription starts to fail, suggesting that the splicing event is not the result of failure of gene transcription. The opposing functions of full-length Mcl-1 and Mcl- $1_{\text {Exon-1 }}$ were confirmed by transfection of U937 cells, illustrating that differential splicing of the Mcl-1 gene is a key event in regulating susceptibility of macrophages to apoptosis during infection. Thus, bacterial infection is a stimulus for alternative splicing of Bcl-2 family genes.

The importance of tightly regulating the onset of apoptosis induction in macrophage-mediated antibacterial defense. The dynamic regulation of Mcl-1 isoform expression apparently determines the timing of MMP and the switch in macrophage susceptibility to apoptosis during pneumococcal infection. The importance of this pathway to the host is illustrated by the variety of strategies employed by bacteria to subvert this process, for instance, by initiating apoptosis downstream of MMP (42). Bacteria may induce macrophage apoptosis in the first few hours after exposure, killing the macrophages before key host defense functions are completed (43). Although these forms of pathogen-mediated apoptosis most often involve direct caspase activation, induction of Mcl-1 degradation by Shiga toxin has been described in endothelial cells (44). In contrast, $M$. tuberculosis delays host-mediated macrophage apoptosis to prolong intracellular survival (45). Although a variety of mechanisms, including modulation of death receptor signaling, have been implicated in this process (45), evidence of upregulation of antiapoptotic Bcl-2 proteins, including Mcl-1 and A1, has been reported $(16,41)$. Inhibition of MMP in macrophages during bacterial infection has also been identified as a mechanism by which intracellular bacteria ensure prolonged survival (42).

Mcl-1 probably also contributes to the regulation of apoptosis in neutrophils during infection, as it has been shown to mediate apoptosis inhibition by GM-CSF, IL-1 $\beta$, or bacterial lipopolysaccharide (46). Furthermore, proteasome inhibition delays neutrophil apoptosis in association with decreased turnover of Mcl-1 (47). Upregulation of Mcl-1 can delay apoptosis in neutrophils during Toxoplasma gondii infection, demonstrating its role in prolonging survival of phagocytes engaged in critical host defense functions (48). In view of the important role of neutrophils in successful resolution of pneumococcal pneumonia (49), it is likely that levels of Mcl-1 expression in neutrophils also contribute to the outcome of pneumococcal infection.
Although altered Mcl-1 expression in cells during exposure to microorganisms has been described previously $(16,27,44,48)$, the functional consequences of altered $\mathrm{Mcl}-1$ expression in vivo during the host response to infection have not previously been demonstrated. We demonstrate that delaying the induction of apoptosis by overexpressing Mcl-1 in macrophages from Mcl-1 transgenic mice reduces AM apoptosis as well as the efficiency of bacterial clearance in a subclinical model of pneumococcal infection. Because this model is not associated with significant recruitment of neutrophils and AMs are the major source of apoptotic cells in this model (18), it is likely that AMs - and by extension, AM apoptosis - indeed play a critical role in antibacterial host defense. Thus, inhibition of AM apoptosis by 2 different experimental systems, namely overexpression of Mcl-1 (this study) and caspase inhibition (18), is associated with an increased likelihood of bacteremia, suggesting that AM apoptosis has an important role in preventing invasive pneumococcal disease.

Factors activated during apoptosis induction could also contribute to antibacterial host defense by directly killing bacteria. Caspase activation occurs downstream of Mcl-1 downregulation and MMP in macrophages following pneumococcal infection. The identity of the caspases involved requires delineation, but experiments with caspase-1-deficient mice suggest that caspase-1 does not play a role (H.M. Marriott and D.H. Dockrell, unpublished data). Loss of MMP has been associated with increased ROS and NO production, which could contribute to bacterial killing and/or provide an additional stimulus for caspase activation (50). These considerations are important in optimizing the use of experimental therapies such as NO synthase and caspase inhibitors $(51,52)$. Although they may improve survival in models of overwhelming infection, they could worsen the outcome in subclinical infection by delaying the apoptosis phase necessary for optimal bacterial killing (18).

Conclusion. We provide evidence that pneumococcal-associated macrophage apoptosis represents a host defense that is tightly regulated by the dynamic alteration and pattern of Mcl-1 isoform expression. Upregulation of full-length Mcl-1 initially maintains macrophage viability, while subsequent downregulation of full-length Mcl-1 and upregulation of a proapoptotic isoform,

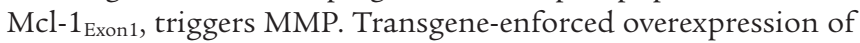
Mcl-1 delays the onset of apoptosis induction and impedes macrophage-mediated bacterial clearance.

\section{Methods}

Bacteria. Type 1 S. pneumoniae (WHO reference laboratory strain, SSISP; Statens Seruminstitut) were grown as previously described and opsonized in RPMI (Sigma-Aldrich) containing 10\% anti-pneumococcal immune serum (22). In experiments involving murine cells, bacteria were opsonized in serum from immunized mice with detectable antibodies against type 1 S. pneumoniae (53). For in vivo infection, type 2 S. pneumoniae (D39 strain) were used (18).

Cells and infection. Human PBMCs were isolated from whole blood donated by healthy volunteers as previously described with informed consent as approved by the South Sheffield Regional Ethics Committee of Royal Hallamshire Hospital (Sheffield, United Kingdom) (22). Murine BMDMs were obtained by culturing marrow from mice expressing human Mcl-1 as a transgene or from nontransgenic littermates (10). Marrow cells were plated at $0.5 \times 10^{6}$ cells $/ \mathrm{ml}$ and cultured as described in DMEM (SigmaAldrich) containing 10\% FCS and 10\% L929 conditioned medium (18). At 14 days, representative wells were scraped to determine the concentration of BMDMs. U937 cells were cultured in RPMI plus 10\% FCS, 2 mM GlutaMAX (Invitrogen Corp.), $10 \mathrm{mM}$ HEPES (Invitrogen Corp.), $1 \mathrm{mM}$ sodium pyruvate (Invitrogen Corp.) and $4.5 \mathrm{~g} / \mathrm{l}$ of glucose (Sigma-Aldrich). MDM 
or BMDM cultured for 14 days or U937 cells were infected with opsonized S. pneumoniae at a MOI of 10 or mock-infected (22). In certain experiments, $10 \mu \mathrm{M}$ MG-132 (Calbiochem) as a proteasome inhibitor; $50 \mu \mathrm{M}$ zVADfmk (Enzymes Systems Products) as a pan-caspase inhibitor; $N$-benzyloxycarbonyl-Phe-Ala fluoromethyl ketone (Enzyme Systems Products) as a control for zVADfmk; $50 \mu \mathrm{M}$ ALLN (Calbiochem) as a calpain I and II inhibitor; $50 \mu \mathrm{M}$ Cathepsin Inhibitor I (Calbiochem) as a cathepsin B, L, and S, and papain inhibitor; or complete protease inhibitor cocktail (Roche) as a broad spectrum serine and cysteine protease inhibitor was added 1 hour before infection and after washing at 4 hours. Actinomycin D (5 $\mu \mathrm{g} / \mathrm{ml}$; SigmaAldrich) was added 4 hours after infection. For UV irradiation, MDMs were exposed to $120 \mathrm{~mJ} / \mathrm{cm}^{2}$ of irradiation (Stratalinker 1800; Stratagene).

Generation of MCL-1 expression constructs. Expression constructs containing $\mathrm{Mcl}-1_{\mathrm{WT}}$ and $\mathrm{Mcl}-1_{\mathrm{S} / \Delta \mathrm{TM}}$ sequences, pcDNA Bax, and insertless PCR3.1 vector were as described (14). For generation of the PCR3.1 Mcl-1 $1_{\text {Exon } 1}$ construct, the following primer pairs were used: Mcl-1 Kozak F 5'-GCCACCATGTTTGGCCTCAAAAGAAACGCG-3', Mcl-1 EX1R 5'-GAATTCTTAATGAACCCGCTTACCTTG- $3^{\prime}$, with genomic DNA as template. The resulting fragments were excised from agarose gels, and the DNA was extracted using the QIAEXII Gel Extraction Kit (Qiagen) and directly cloned into pCR3.1 TOPO (Invitrogen Corp.).

SDS-PAGE and Western immunoblotting. Whole-cell extracts and cytosolic and membrane fractions were extracted as described (34). For detection of AIF in nuclear fractions, cells were lysed in buffer containing $20 \mathrm{mM}$ HEPES, $10 \mathrm{mM} \mathrm{KCl}, 1.5 \mathrm{mM} \mathrm{MgCl}$, 1 mM EDTA, 1 mM EGTA, $1 \mathrm{mM}$ DTT, $250 \mathrm{mM}$ sucrose, and protease inhibitor cocktail as described (54). The pellet was resuspended in lysis buffer (nuclear fraction). The supernatant was spun at $10,000 \mathrm{~g}$ for 15 minutes at $4^{\circ} \mathrm{C}$. The pellet was resuspended in lysis buffer (mitochondrial fraction); the supernatant was spun at $500,000 \mathrm{~g}$ for 1 hour at $4^{\circ} \mathrm{C}$, and the resulting supernatant was used as the cytosolic fraction. Protein was quantified using a modified Lowry protocol ( $D C$ protein assay; Bio-Rad Laboratories), and equal protein was loaded per lane, either $25 \mu \mathrm{g}$ of total protein or $10 \mu \mathrm{g}$ of protein from cell fractions. Samples were separated by SDS-PAGE (12\% or $15 \%)$ and blotted onto nitrocellulose membranes (Bio-Rad Laboratories) with protein transfer confirmed by Ponceau S staining. Blots were incubated overnight at $4{ }^{\circ} \mathrm{C}$ with antibodies against Mcl-1 (rabbit polyclonal SC-19, 1:500; Santa Cruz Biotechnology Inc. or rabbit polyclonal, 1:1,000; BD Biosciences), cytochrome $c$ (mouse monoclonal, clone 7H8.2C12, 1:1,000; BD Biosciences), Bcl- $\mathrm{x}_{\mathrm{L}}$ (rabbit polyclonal, 1:1,000; BD Biosciences), p53 (mouse monoclonal, clone DO-1; Santa Cruz Biotechnology Inc.), actin (goat polyclonal, 1:500; Santa Cruz Biotechnology Inc. or rabbit polyclonal, 1:2,000; Sigma-Aldrich), GAPDH (mouse monoclonal, 1:2,000; Chemicon), Histone H1 (AE-4, mouse monoclonal, 1:500; Santa Cruz Biotechnology Inc.) or AIF $(1: 1,000)$ (19). Protein detection was done with HRP-conjugated secondary antibodies (Dako) and ECL (Amersham Pharmacia). Bands were quantified using Image J 1.32 software $(\mathrm{NIH})$, and after confirming equivalence of Ponceau S staining for each time point, Mcl-1 bands were compared with the mean of the mock-infected bands for that donor.

In vitro translation. To prepare $\mathrm{Mcl}-1$ proteins, a coupled in vitro transcription/translation system (Promega) was used in accordance with the manufacturer's instructions using Mcl-1 constructs in pCR3.1.

RNase protection assay. Total RNA was harvested from MDMs cultured in $25-\mathrm{cm}^{2}$ plastic flasks using guanidine isothiocyanate solution (4 $\mathrm{M}$ guanidine isothiocyanate, $5 \mathrm{mM}$ sodium citrate [pH 7.0], $0.1 \mathrm{M}$ $\beta$-mercaptoethanol [all Sigma-Aldrich]) and $\mathrm{CsCl}$ as previously described (55).
Assays were performed using $5 \mathrm{mg}$ of RNA and the APO-2b probe set according to the RiboQuant protocol (BD Biosciences).

Apoptosis detection. Nuclear morphology was examined on all cells by DAPI (Molecular Probes) as previously reported (22). Apoptosis was estimated by blinded reviewers who counted 300 cells per coverslip in duplicate samples for the presence of condensed or fragmented nuclei. To detect loss of $\Delta \psi_{\mathrm{m}}$, cells were stained in $250 \mu \mathrm{L}$ RPMI containing $10 \mu \mathrm{M}$ 5,5',6,6'-tetrachloro$1,1^{\prime}, 3,3^{\prime}$-tetraethylbenzimidazolocarbocyanine iodide (JC-1) (Molecular probes) and $2 \mu \mathrm{M}$ Hoechst 33342 (Calbiochem) as a nuclear counterstain for 30 minutes at $37^{\circ} \mathrm{C}$. Coverslips were mounted and examined by fluorescent microscopy. Loss of $\Delta \psi_{\mathrm{m}}$ was confirmed by loss of red punctate staining. In these experiments, MDMs pretreated with $5 \mu \mathrm{M}$ staurosporine (Sigma-Aldrich) for 2 hours were used as a positive control. To quantify loss of $\Delta \psi_{\mathrm{m}}, 2 \times 10^{6}$ cells were stained with JC- 1 as above, washed, scraped, and analyzed by a FACSCalibur flow cytometer (BD Biosciences - Pharmingen). At least 10,000 cells were analyzed for each experimental condition.

Transient transfection with Mcl-1 isoform constructs to assess effects on cell death. pCR3.1-based vectors $(0.5 \mu \mathrm{g})$ were transiently transfected into U937 cells together with pcDNA Bax as a positive control, and empty PCR3.1 vector as a negative control; $0.5 \mu \mathrm{g}$ of vector expressing EGFP was cotransfected as a reporter. Transfection was by nucleofection (Amaxa GmbH), in accordance with the manufacturer's protocol, 4 hours after infection. Cytospin preparations were performed 16 hours after transfection, and apoptosis was assessed as above.

Bacterial killing assay. The intracellular bacterial killing assay was performed at 14 hours as previously described (34) with quadruplicate wells lysed for each data point.

In vivo infection. $\mathrm{Mcl}-1$ transgenic mice or nontransgenic littermates were infected by intratracheal instillation of $10^{4}$ or $10^{5} \mathrm{CFU}$ type $2 \mathrm{~S}$. pneumoniae, as described previously (18). Mice were killed 14 hours after infection, and bronchoalveolar lavage and lungs were collected. Bacterial numbers in blood and lung and AM apoptosis, assessed by flow cytometry after Annexin V-PE/ToPro 3 staining, or assessment of nuclear morphology on cytospin preparations were as previously described (18). All animal experiments were conducted in accordance with the Home Office Animals (Scientific Procedures) Act of 1986 and was approved by the animal project review committee of the University of Sheffield.

Statistics. Results are recorded as mean and SEM. Parametric or nonparametric testing was performed with the indicated tests using Prism 4.0 software (GraphPad Inc.). Significance was defined as $P<0.05$.

\section{Acknowledgments}

This work was supported by a Wellcome Trust Advanced Clinical Fellowship to D.H. Dockrell (065054). C.D. Bingle and M.K.B. Whyte were supported by Yorkshire Cancer Research project grant 00/295 and R.W. Craig by NIH grant CA57359 for work on Mcl-1. We appreciate helpful discussion with Jon Sayers and Simon Brown during preparation of this manuscript.

Received for publication March 31, 2004, and accepted in revised form November 30, 2004.

Address correspondence to: David H. Dockrell, Division of Genomic Medicine, F-Floor, University of Sheffield, Beech Hill Road, Sheffield S10 2RX, United Kingdom. Phone: 44-114-2724072; Fax: 44-114-2713892; E-mail: d.h.dockrell@sheffield.ac.uk.
1. Sibille, Y., and Reynolds, H.Y. 1990. Macrophages and polymorphonuclear neutrophils in lung defense and injury. Am. Rev. Respir. Dis. 141:471-501.

2. Liu, H., Perlman, H., Pagliari, L.J., and Pope, R.M.
2001. Constitutively activated Akt-1 is vital for the survival of human monocyte-differentiated macrophages. Role of Mcl-1, independent of nuclear factor (NF)-kappaB, Bad, or caspase activation.
J. Exp. Med. 194:113-126.

3. Zychlinsky, A., and Sansonetti, P. 1997. Apoptosis in bacterial pathogenesis [review]. J. Clin. Invest. 100:493-495. 
4. Molloy, A., Laochumroonvorapong, P., and Kaplan, G. 1994. Apoptosis, but not necrosis, of infected monocytes is coupled with killing of intracellular bacillus Calmette-Guerin. J. Exp. Med. 180:1499-1509.

5. Kozopas, K.M., Yang, T., Buchan, H.L., Zhou, P., and Craig, R.W. 1993. MCL1, a gene expressed in programmed myeloid cell differentiation, has sequence similarity to BCL2. Proc. Natl. Acad. Sci. U. S. A. 90:3516-3520.

6. Yang, T., Kozopas, K.M., and Craig, R.W. 1995. The intracellular distribution and pattern of expression of Mcl-1 overlap with, but are not identical to, those of Bcl-2. J. Cell Biol. 128:1173-1184.

7. Yang, T., Buchan, H.L., Townsend, K.J., and Craig, R.W. 1996. MCL-1, a member of the BLC-2 family, is induced rapidly in response to signals for cell differentiation or death, but not to signals for cell proliferation. J. Cell. Physiol. 166:523-536.

8. Zhou, P., Qian, L., Kozopas, K.M., and Craig, R.W. 1997. Mcl-1, a Bcl-2 family member, delays the death of hematopoietic cells under a variety of apoptosis-inducing conditions. Blood. 89:630-643.

9. Chao, J.R., et al. 1998. mcl-1 is an immediate-early gene activated by the granulocyte-macrophage colony-stimulating factor (GM-CSF) signaling pathway and is one component of the GM-CSF viability response. Mol. Cell. Biol. 18:4883-4898.

10. Zhou, P., et al. 1998. Mcl-1 in transgenic mice promotes survival in a spectrum of hematopoietic cell types and immortalization in the myeloid lineage. Blood. 92:3226-3239.

11. Green, D.R., and Reed, J.C. 1998. Mitochondria and apoptosis. Science. 281:1309-1312

12. Cuconati, A., Mukherjee, C., Perez, D., and White, E. 2003. DNA damage response and MCL-1 destruction initiate apoptosis in adenovirus-infected cells. Genes Dev. 17:2922-2932.

13. Nijhawan, D., et al. 2003. Elimination of Mcl-1 is required for the initiation of apoptosis following ultraviolet irradiation. Genes Dev. 17:1475-1486.

14. Bingle, C.D., et al. 2000. Exon skipping in Mcl-1 results in a bcl-2 homology domain 3 only gene product that promotes cell death. J. Biol. Chem. 275:22136-22146

15. Bae, J., Leo, C.P., Hsu, S.Y., and Hsueh, A.J. 2000 MCL-1S, a splicing variant of the antiapoptotic BCL-2 family member MCL-1, encodes a proapoptotic protein possessing only the $\mathrm{BH} 3$ domain. J. Biol. Chem. 275:25255-25261.

16. Sly, L.M., Hingley-Wilson, S.M., Reiner, N.E., and McMaster, W.R. 2003. Survival of Mycobacterium tuberculosis in host macrophages involves resistance to apoptosis dependent upon induction of antiapoptotic Bcl-2 family member Mcl-1. J. Immunol. 170:430-437.

17. Ali, F., et al. 2003. Streptococcus pneumoniae-associated human macrophage apoptosis after bacterial internalization via complement and Fcgamma receptors correlates with intracellular bacterial load. J. Infect. Dis. 188:1119-1131.

18. Dockrell, D.H., et al. 2003. Alveolar macrophage apoptosis contributes to pneumococcal clearance in a resolving model of pulmonary infection. J. Immunol. 171:5380-5388.

19. Susin, S.A., et al. 1999. Molecular characterization of mitochondrial apoptosis-inducing factor. Nature. 397:441-446.

20. Braun, J.S., et al. 2001. Apoptosis-inducing factor mediates microglial and neuronal apoptosis caused by pneumococcus. J. Infect. Dis. 184:1300-1309.

21. Michels, J., et al. 2004. Mcl-1 is required for Akata6 B-lymphoma cell survival and is converted to a cell death molecule by efficient caspase-mediated cleavage. Oncogene. 23:4818-4827.
22. Dockrell, D.H., Lee, M., Lynch, D.H., and Read, R.C. 2001. Immune-mediated phagocytosis and killing of Streptococcus pneumoniae are associated with direct and bystander macrophage apoptosis. J. Infect. Dis. 184:713-722.

23. Underhill, D.M., and Ozinsky, A. 2002. Phagocytosis of microbes: complexity in action. Annu. Rev. Immunol. 20:825-852.

24. Gordon, S.B., Irving, G.R., Lawson, R.A., Lee, M.E., and Read, R.C. 2000. Intracellular trafficking and killing of Streptococcus pneumoniae by human alveolar macrophages are influenced by opsonins. Infect. Immun. 68:2286-2293.

25. Rojas, M., Barrera, L.F., Puzo, G., and Garcia, L.F. 1997. Differential induction of apoptosis by virulent Mycobacterium tuberculosis in resistant and susceptible murine macrophages: role of nitric oxide and mycobacterial products. J. Immunol. 159:1352-1361

26. Keane, J., et al. 1997. Infection by Mycobacterium tuberculosis promotes human alveolar macrophage apoptosis. Infect. Immun. 65:298-304.

27. Doran, K.S., Liu, G.Y., and Nizet, V. 2003. Group B streptococcal $\beta$-hemolysin/cytolysin activates neutrophil signaling pathways in brain endothelium and contributes to development of meningitis. J. Clin. Invest. 112:736-744. doi:10.1172/ JCI200317335.

28. Ulett, G.C., Bohnsack, J.F., Armstrong, J., and Adderson, E.E. 2003. Beta-hemolysin-independent induction of apoptosis of macrophages infected with serotype III group B streptococcus. J. Infect. Dis. 188:1049-1053.

29. Hockenbery, D.M., Oltvai, Z.N., Yin, X.M., Milliman, C.L., and Korsmeyer, S.J. 1993. Bcl-2 functions in an antioxidant pathway to prevent apoptosis. Cell. 75:241-251.

30. Vieira, H.L., et al. 2001. The adenine nucleotide translocator: a target of nitric oxide, peroxynitrite, and 4-hydroxynonenal. Oncogene. 20:4305-4316.

31. Vazquez-Torres, A., Jones-Carson, J., Mastroeni, P., Ischiropoulos, H., and Fang, F.C. 2000. Antimicrobial actions of the NADPH phagocyte oxidase and inducible nitric oxide synthase in experimental salmonellosis. I. Effects on microbial killing by activated peritoneal macrophages in vitro. J. Exp. Med. 192:227-236

32. Murdock, D.G., Boone, B.E., Esposito, L.A., and Wallace, D.C. 1999. Up-regulation of nuclear and mitochondrial genes in the skeletal muscle of mice lacking the heart/muscle isoform of the adenine nucleotide translocator. J. Biol. Chem. 274:14429-14433.

33. Li, C.Q., Trudel, L.J., and Wogan, G.N. 2002. Nitric oxide-induced genotoxicity, mitochondrial damage, and apoptosis in human lymphoblastoid cells expressing wild-type and mutant p53. Proc. Natl. Acad. Sci. U. S. A. 99:10364-10369.

34. Marriott, H.M., et al. 2004. Nitric oxide levels regulate macrophage commitment to apoptosis or necrosis during pneumococcal infection. FASEB J. 18:1126-1128

35. Beltran, B., Mathur, A., Duchen, M.R., Erusalimsky, J.D., and Moncada, S. 2000. The effect of nitric oxide on cell respiration: A key to understanding its role in cell survival or death. Proc. Natl. Acad. Sci. U. S. A. 97:14602-14607.

36. Ban, J., Eckhart, L., Weninger, W., Mildner, M., and Tschachler, E. 1998. Identification of a human cDNA encoding a novel $\mathrm{Bcl}-\mathrm{x}$ isoform. Biochem. Biophys. Res. Commun. 248:147-152.

37. Korsmeyer, S.J., et al. 2000. Pro-apoptotic cascade activates BID, which oligomerizes BAK or BAX into pores that result in the release of cytochrome c. Cell Death Differ. 7:1166-1173.
38. Letai, A., et al. 2002. Distinct BH3 domains either sensitize or activate mitochondrial apoptosis, serving as prototype cancer therapeutics. Cancer Cell. 2:183-192.

39. O'Connor, L., et al. 1998. Bim: a novel member of the $\mathrm{Bcl}-2$ family that promotes apoptosis. EMBO J. 17:384-395.

40. Fang, W., Rivard, J.J., Mueller, D.L., and Behrens, T.W. 1994. Cloning and molecular characterization of mouse bcl-x in B and T lymphocytes. J. Immunol. 153:4388-4398

41. Kausalya, S., Somogyi, R., Orlofsky, A., and Prystowsky, M.B. 2001. Requirement of A1-a for bacillus Calmette-Guerin-mediated protection of macrophages against nitric oxide-induced apoptosis. J. Immunol. 166:4721-4727.

42. Gao, L.Y., and Kwaik, Y.A. 2000. The modulation of host cell apoptosis by intracellular bacterial pathogens. Trends Microbiol. 8:306-313.

43. Navarre, W.W., and Zychlinsky, A. 2000. Pathogeninduced apoptosis of macrophages: a common end for different pathogenic strategies. Cell Microbiol. 2:265-273.

44. Erwert, R.D., et al. 2003. Shiga toxin induces decreased expression of the anti-apoptotic protein Mcl-1 concomitant with the onset of endothelial apoptosis. Microb. Pathog. 35:87-93.

45. Kornfeld, H., Mancino, G., and Colizzi, V. 1999. The role of macrophage cell death in tuberculosis. Cell Death Differ. 6:71-78.

46. Moulding, D.A., Quayle, J.A., Hart, C.A., and Edwards, S.W. 1998. Mcl-1 expression in human neutrophils: regulation by cytokines and correlation with cell survival. Blood 92:2495-2502.

47. Derouet, M., Thomas, L., Cross, A., Moots, R.J., and Edwards, S.W. 2004. Granulocyte macrophage colony-stimulating factor signaling and proteasome inhibition delay neutrophil apoptosis by increasing the stability of Mcl-1. J. Biol. Chem. 279:26915-26921.

48. Channon, J.Y., Miselis, K.A., Minns, L.A., Dutta, C., and Kasper, L.H. 2002. Toxoplasma gondii induces granulocyte colony-stimulating factor and granulocyte-macrophage colony-stimulating factor secretion by human fibroblasts: implications for neutrophil apoptosis. Infect. Immun. 70:6048-6057

49. Haslett, C. 1999. Granulocyte apoptosis and its role in the resolution and control of lung inflammation. Am. J. Respir. Crit. Care Med. 160:S5-S11.

50. Zorov, D.B., Filburn, C.R., Klotz, L.O., Zweier, J.L., and Sollott, S.J. 2000. Reactive oxygen species (ROS)-induced ROS release: a new phenomenon accompanying induction of the mitochondrial permeability transition in cardiac myocytes. J. Exp. Med. 192:1001-1014.

51. Bergeron, Y., Ouellet, N., Simard, M., Olivier, M., and Bergeron, M.G. 1999. Immunomodulation of pneumococcal pulmonary infection with $\mathrm{N}(\mathrm{G})$ monomethyl-L-arginine. Antimicrob. Agents Chemother. 43:2283-2290.

52. Hotchkiss, R.S., et al. 2000. Caspase inhibitors improve survival in sepsis: a critical role of the lymphocyte. Nat. Immunol. 1:496-501.

53. Dullforce, P., Sutton, D.C., and Heath, A.W. 1998. Enhancement of T cell-independent immune responses in vivo by CD40 antibodies. Nat. Med. 4:88-91.

54. Qiu, J.H., et al. 2000. Proteasome inhibitors induce cytochrome c-caspase-3-like protease-mediated apoptosis in cultured cortical neurons. J. Neurosci. 20:259-265.

55. Bingle, C.D. 1996. Measurement of anti-oxidant gene expression. In Free radicals: a practical approach. N.A. Punchard and F.J. Kelly, editors. Oxford University Press. Oxford, United Kingdom. 287-300. 